\title{
Dimensões da patela relacionadas à massa corporal do cão para emprego na confecção de prótese: estudo em cadáver
}

[Patellar dimensions related to canine body mass for use in the manufacture of prosthesis: cadaver study]

\section{B.S. Okano $^{1}$, S.J. Colorado ${ }^{1}$, L.R. Drummond ${ }^{2,3}$, J.A.C. Varon $^{1,4}$, E.O. Carmona ${ }^{1}$, A. A. Corteze ${ }^{1}$, I.D.M. Rodriguez ${ }^{5}$, C.M.F. Rezende 6}

${ }^{1}$ Aluno de pós-graduação - Escola de Veterinária - Universidade Federal de Minas Gerais - Belo Horizonte, MG

${ }^{2}$ Aluno de pós-graduação - Instituto de Ciências Biológicas - Universidade Federal de Minas Gerais - Belo Horizonte, MG

${ }^{3}$ Universidade do Estado de Minas Gerais - Divinópolis, MG

${ }^{4}$ Universidad Cooperativa de Colombia - Bucaramanga, Colômbia

${ }^{5}$ Facultad de Medicina Veterinária y Zootecnia - Universidade Del Tolima - Tolima, Colômbia

${ }^{6}$ Escola de Veterinária - Universidade Federal de Minas Gerais - Belo Horizonte, MG

\section{RESUMO}

O objetivo deste trabalho foi mensurar as dimensões da patela de cadáveres caninos e avaliar sua relação com a massa corporal. Para a realização das medidas patelares, foram utilizados 70 cadáveres de cães adultos, com massa corpórea de 1 a $50 \mathrm{~kg}$, sem evidência clínica de afecção na articulação femorotibiopatelar. Com auxílio de um paquímetro, foram mensurados os comprimentos externo e interno, a largura externa no terço médio, a largura e a espessura internas nos terços proximal, médio e distal das patelas. Também foram mensuradas a largura nos terços proximal, médio e distal, a profundidade nos terços proximal, médio e distal do sulco troclear; essas medidas foram exclusivamente internas. Observou-se, de forma geral, forte correlação entre as medidas patelares e a massa corporal de cadáveres de cães. Nas condições deste estudo, pode-se concluir que o tamanho da patela varia segundo a massa corporal do cão e que essas medidas devem ser consideradas ao se planejar uma substituição protética.

Palavras-chave: anatomia patelar, prótese patelar, luxação de patela

\begin{abstract}
The aim of this study was to measure the canine cadaver patellar dimensions and evaluate its relationship with body mass. 70 cadavers of adult dogs were used, with a body mass between 1 and 50kg, without clinical evidence of affection in the stifle joint. The external and internal lengths, external width in the middle third, width and thickness of the proximal, middle and distal thirds were measured using a pachymeter. The width was also measured in the proximal third, middle third and distal third, depth in the proximal third, middle third and distal third of the trochlear groove; these measurements were exclusively internal. A strong correlation was observed between the patellar dimension and body mass of canine cadavers. Under this study conditions, it is possible to conclude that the patellar size varies according to the canine body mass and these measures should be considered when planning a prosthetic replacement.
\end{abstract}

Keywords: patellar anatomy, patellar prosthesis, patellar luxation

\section{INTRODUÇÃO}

A patela é o maior osso sesamoide do cão e está localizada no tendão de inserção do músculo quadríceps femoral, sendo o ponto central de fixação do tendão do quadríceps e do ligamento patelar; juntamente com a tróclea femoral, forma a articulação femoropatelar (Evans e Lahunta, 2013; Liebich et al., 2016). Ela é um componente fundamental do mecanismo extensor do quadríceps, responsável por direcionar as forças do quadríceps femoral ao ligamento

Recebido em 15 de abril de 2018

Aceito em 15 de abril de 2019

E-mail: barbaraokano@gmail.com 
patelar, proteger o tendão do quadríceps da fricção e aumentar a superfície de deslizamento do tendão (Carpenter e Cooper, 2000). O alinhamento da patela, do músculo quadríceps, do ligamento patelar e da tróclea impede a luxação ou subluxação da patela (Mckee e Cook, 2006; Rezende et al., 2016).

A luxação de patela (LP) é uma das condições ortopédicas mais comuns que acomete a articulação femorotibiopatelar dos cães. Pode resultar em dor, claudicação, osteoartrose e impotência funcional do membro (Roush, 1993; LaFond et al., 2002; Kowaleski et al., 2012). Em um estudo de avaliação da epidemiologia da LP, encontrou-se que $1,3 \%$ do total de casos atendidos em hospitais da Inglaterra (O’Neill et al., 2016) era de LP. Lara (2011) avaliou as lesões encontradas no transoperatório de 252 cães com LP e observou lesões na cartilagem da patela em $55,9 \%$ dos casos.

As técnicas cirúrgicas para a correção da LP e o alinhamento do quadríceps resumem-se em reconstrução dos tecidos moles e na reconstrução óssea (Piermattei et al., 2006). Os resultados das cirurgias de correção da LP são considerados insatisfatórios, pois $25 \%$ a $50 \%$ dos pacientes não têm retorno da função completa do membro (Willauer e Vasseur, 1987; Daems et al., 2009). Gareth e Sorrel (2006) relatam que a frequência de complicações pós-operatórias é de $18 \%$, e em $2,3 \%$ dos animais estudados a cirurgia não corrigiu a LP, mesmo após dois procedimentos cirúrgicos. Daems e colaboradores (2009) acreditam que um dos motivos da instabilidade patelar após a correção cirúrgica é a presença de lesões na patela.

Para o tratamento de lesões graves da superfície articular da patela, de perda da convexidade e de traumatismos irreparáveis, a literatura recomenda a patelectomia (Piermattei et al., 2006). Entretanto, essa técnica não melhora a função do membro, o alinhamento e a estabilidade femoropatelar (Campbell e Pond, 1972; Roush, 1993). A prótese de patela para cães seria uma alternativa para o tratamento de lesões irreparáveis da patela, no entanto, em medicina veterinária, ainda não existe essa prótese disponível no mercado. Para a confecção de um protótipo de prótese, é necessário o conhecimento anatômico da patela e de suas medidas.
Até o presente momento, algumas medidas da patela foram estudadas para a avaliação de deformidades ósseas em casos de LP (Towle et al., 2005; Yasukawa et al., 2016), porém elas são insuficientes para a confecção de prótese de patela. Ainda não foi estudada também a relação entre as medidas patelares e o porte do cão. Portanto, o objetivo deste trabalho é determinar as medidas da patela de cães e sua relação com a massa corporal, com vistas a propiciar informações iniciais básicas para a elaboração futura de prótese de patela.

\section{MATERIAL E MÉTODOS}

O uso de cadáver para fins científicos dispensa a aprovação da Comissão de Ética no Uso de Animais da Universidade Federal de Minas Gerais (Ofício Circular Ceua 001/2017). No presente trabalho, foram utilizados 70 cadáveres de cães adultos, independentemente de raça ou sexo, com massa corpórea de um a $50 \mathrm{~kg}$, sem evidência clínica de afecção da articulação femorotibiopatelar para realização do estudo das medidas da patela em cadáver.

Foram utilizadas as patelas dos membros pélvicos direito e esquerdo de 70 cadáveres, totalizando 140 patelas. Realizou-se a média entre as mesmas medidas dos membros direito e esquerdo de cada animal, portanto o $\mathrm{n}$ amostral do experimento foi de 70 amostras. Os cadáveres foram divididos em grupo 1 ( 1 a $4,99 \mathrm{~kg}$ ); grupo 2 (5 a 9,99kg); grupo 3 (10 a 19,99kg); grupo 4 (20 a 29,99kg) e grupo 5 (30 a $50 \mathrm{~kg})$, sendo 14 cadáveres por grupo.

Todas as mensurações foram realizadas pelo mesmo avaliador, utilizando-se um paquímetro analógico (Brasfort ${ }^{\circledR}$, Brasília, Brasil; 150mm) com precisão de $0,01 \mathrm{~mm}$. As medidas foram tomadas inicialmente da patela recoberta pelos tecidos e foram denominadas medidas externas. Após artrotomia, tomaram-se as medidas diretamente da patela, consideradas medidas internas. As mensurações externas da patela foram comprimento e largura no terço médio. Para mensuração interna, a patela foi dividida em terço proximal, médio e distal (Fig. 1A). As mensurações internas da patela foram largura e espessura nos terços proximal, médio e distal e comprimento da patela (Fig. 1C). Assim como na divisão da patela, a tróclea foi dividida em terços 


\section{Dimensões da patela...}

proximal, médio e distal (Fig. 1B). Essas delimitações foram utilizadas para permitir que as medidas de largura e profundidade do sulco fossem realizadas no mesmo local (Fig. 1D). As mensurações do sulco troclear foram exclusivamente internas e compostas por largura nos terços proximal, médio e distal, profundidade nos terços proximal, médio e distal. As três medidas de profundidade foram mensuradas tanto no côndilo lateral como no medial.

Os dados referentes às medidas patelares foram apresentados como médiaterro-padrão da média.
A normalidade dos dados foi verificada por meio do teste de Shapiro-Wilk. As diferenças entre os grupos foram analisadas por meio de ANOVA One Way, seguida do post hoc de Tukey. Para a avaliação da relação entre as medidas da patela e a massa corporal, foi utilizada a correlação de Pearson. Para determinação das equações para estimativa de medidas internas da patela com base nas medidas externas, foi utilizada análise de regressão linear. O nível de significância adotado foi de $5 \%$.

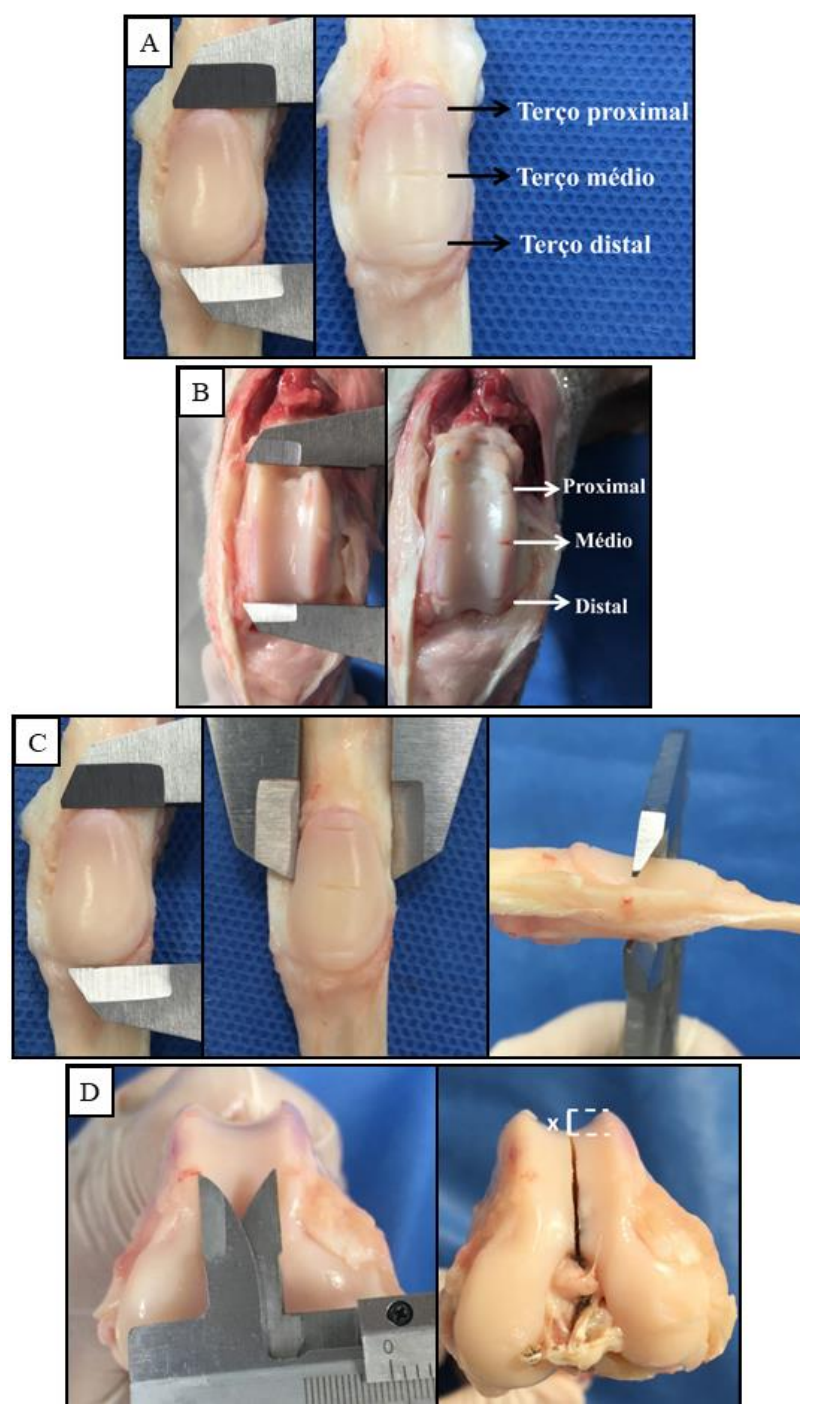

Figura 1. Figuras representativas das medidas patelares e trocleares. Figuras representativas da divisão da patela nos diferentes terços (painel A), da divisão da tróclea nos diferentes terços (B), das medidas de comprimento, largura e espessura da patela (painel C), das medidas de largura e profundidade da tróclea (painel D). Fonte: Arquivo pessoal da autora. 


\section{RESULTADOS E DISCUSSÃO}

A Tab. 1 mostra os dados da mensuração externa do comprimento e da largura da patela nos grupos G1, G2, G3, G4 e G5. Não houve diferença significativa no comprimento externo da patela entre os grupos G1 e G2. Os grupos G3 e G4 foram significativamente diferentes dos grupos G1 e G2, mas não entre si. O G5 apresentou diferença significativa quando comparado aos grupos G1, G2 e G3, mas não em relação ao G4. Houve uma correlação forte e positiva entre a massa corporal dos animais e o comprimento externo da patela $(r=0,75$; $\mathrm{P}<0,001)$.
Não houve diferença significativa na largura externa da patela entre os grupos $\mathrm{G} 1$ e G2. O grupo G3 foi significativamente diferente dos grupos G1 e G2. Os grupos G4 e G5 foram significativamente diferentes dos grupos G1, G2 e G3, mas não entre si. Houve uma correlação forte e positiva entre a massa corporal dos animais e a largura externa da patela $(r=0,82$; $\mathrm{P}<0,001)$. A Tab. 2 mostra a média das medidas internas da patela nos grupos G1, G2, G3, G4 e G5. Com relação ao comprimento interno da patela, houve diferença significativa entre todos os grupos. Houve uma correlação forte e positiva entre a massa corporal dos animais e o comprimento interno da patela $(\mathrm{r}=0,86$; $\mathrm{P}<0,001)$

Tabela 1. Média das medidas externas da patela de cadáveres de cães nos grupos G1, G2, G3, G4 e G5

\begin{tabular}{lccccc}
\hline \multirow{2}{*}{ Mensurações externas } & \multicolumn{5}{c}{ Medidas (cm) } \\
\cline { 2 - 6 } & $\mathrm{G} 1$ & $\mathrm{G} 2$ & $\mathrm{G} 3$ & $\mathrm{G} 4$ & $\mathrm{G} 5$ \\
\hline Comprimento externo da patela & $1,39 \pm 0,04$ & $1,65 \pm 0,08$ & $2,08 \pm 0,06^{* \#}$ & $2,28 \pm 0,09^{* \#}$ & $2,50 \pm 0,07^{* \#+}$ \\
Largura externa da patela & $0,81 \pm 0,03$ & $0,91 \pm 0,03$ & $1,22 \pm 0,03^{* \#}$ & $1,42 \pm 0,07^{* \#+}$ & $1,51 \pm 0,04^{* \#+}$ \\
\hline
\end{tabular}

Dados expressos como média \pm EPM. * $\mathrm{P}<0,05$ vs. G1; \# $\mathrm{P}<0,05$ vs. G2; + $\mathrm{P}<0,05$ vs. G3; § $\mathrm{P}<0,05$ vs. G4. $\uparrow$, aumento.

Tabela 2. Média das medidas internas da patela de cadáveres de cães nos grupos G1, G2, G3, G4 e G5

\begin{tabular}{|c|c|c|c|c|c|}
\hline \multirow{2}{*}{ Mensurações internas } & \multicolumn{5}{|c|}{ Medidas $(\mathrm{cm})$} \\
\hline & G1 & G2 & G3 & G4 & G5 \\
\hline Comprimento da patela & $0,93 \pm 0,02$ & $1,13 \pm 0,04^{*}$ & $1,48 \pm 0,06^{* \#}$ & $1,75 \pm 0,04^{* \#+}$ & $1,95 \pm 0,04^{* \#+\S}$ \\
\hline $\begin{array}{l}\text { Largura no terço proximal da } \\
\text { patela }\end{array}$ & $0,48 \pm 0,01$ & $0,56 \pm 0,01 *$ & $0,68 \pm 0,02 * \#$ & $0,82 \pm 0,04^{* \#+}$ & $0,87 \pm 0,02 * \#+\S$ \\
\hline $\begin{array}{l}\text { Largura no terço médio da } \\
\text { patela }\end{array}$ & $0,68 \pm 0,01$ & $0,77 \pm 0,02$ & $0,94 \pm 0,03 * \#$ & $1,04 \pm 0,04 * \#$ & $1,23 \pm 0,03 * \#+\S$ \\
\hline $\begin{array}{l}\text { Largura no terço distal da } \\
\text { patela }\end{array}$ & $0,62 \pm 0,02$ & $0,74 \pm 0,02$ & $0,89 \pm 0,04$ *\# & $1,05 \pm 0,04^{* \#+}$ & $1,21 \pm 0,04 * \#+\S$ \\
\hline $\begin{array}{l}\text { Espessura no terço proximal da } \\
\text { patela }\end{array}$ & $0,43 \pm 0,01$ & $0,52 \pm 0,01$ & $0,67 \pm 0,02 * \#$ & $0,86 \pm 0,02$ *\#+ & $0,98 \pm 0,03 * \#+\S$ \\
\hline $\begin{array}{l}\text { Espessura no terço médio da } \\
\text { patela }\end{array}$ & $0,47 \pm 0,01$ & $0,58 \pm 0,02$ & $0,76 \pm 0,02$ *\# & $0,92 \pm 0,03^{* \#+}$ & $1,03 \pm 0,04 * \#+$ \\
\hline $\begin{array}{l}\text { Espessura no terço distal da } \\
\text { patela }\end{array}$ & $0,44 \pm 0,01$ & $0,56 \pm 0,03$ & $0,71 \pm 0,03 * \#$ & $0,90 \pm 0,02 * \#+$ & $1,02 \pm 0,03 * \#+$ \\
\hline
\end{tabular}

Dados expressos como média \pm EPM. * $\mathrm{P}<0,05$ vs. G1; \# $\mathrm{P}<0,05$ vs. G2; + $\mathrm{P}<0,05$ vs. G3; § $\mathrm{P}<0,05$ vs. G4. $\uparrow$, aumento.

Em relação à largura interna no terço proximal, houve diferença significativa entre todos os grupos. Houve uma correlação forte e positiva entre a massa corporal dos animais e a largura no terço proximal da patela $(\mathrm{r}=0,78 ; \mathrm{P}<0,001)$. Para as medidas da largura no terço médio da patela, não houve diferença significativa entre os grupos G1 e G2. Os grupos G3 e G4 foram significativamente diferentes dos grupos G1 e
G2, mas não entre si. O G5 apresentou diferença significativa quando comparado a todos os outros grupos. Houve uma correlação forte e positiva entre a massa corporal dos animais e a largura do terço médio da patela $(\mathrm{r}=0,80 ; \mathrm{P}<0,001)$. As medidas da largura no terço distal não foram significativamente diferentes entre os grupos G1 e G2. Os grupos G3, G4 e G5 foram diferentes dos grupos G1 e G2 e entre si. Houve uma 
correlação forte e positiva entre a massa corporal dos animais e a largura no terço distal da patela $(\mathrm{r}=0,82 ; \mathrm{P}<0,001)$.

Os dados das medidas de largura da patela sugerem que as patelas diferem de tamanho entre os grupos de massa corporal. Portanto, diferentemente das medidas anteriores, cada grupo tem suas medidas específicas, que devem ser consideradas ao se planejar uma prótese para substituição da patela danificada, pois, se uma medida estrutural é diferente, certamente isso mudará a forma global da patela.

As medidas da espessura da patela no terço proximal não foram significativamente diferentes entre os grupos G1 e G2. Os grupos G3, G4 e G5 foram diferentes dos grupos G1 e G2 e entre si. Houve uma correlação forte e positiva entre a massa corporal dos animais e a espessura no terço proximal da patela $(\mathrm{r}=0,87 ; \mathrm{P}<0,001)$. As medidas da espessura da patela no terço médio não foram significativamente diferentes entre os grupos G1 e G2. O grupo G3 foi diferente dos grupos G1 e G2. Os grupos G4 e G5 foram significativamente diferentes dos grupos G1, G2 e G3, mas não entre si. Houve uma correlação forte e positiva entre a massa corporal dos animais e a espessura no terço médio da patela $(\mathrm{r}=0,83 ; \mathrm{P}<0,001)$. As medidas da espessura da patela no terço distal não foram significativamente diferentes entre os grupos G1 e G2. O grupo G3 foi diferente dos grupos G1 e $\mathrm{G} 2$. Os grupos $\mathrm{G} 4$ e $\mathrm{G} 5$ foram significativamente diferentes dos grupos G1, G2 e G3, mas não entre si. Houve uma correlação forte e positiva entre a massa corporal dos animais e a espessura no terço distal da patela $(\mathrm{r}=0,84 ; \mathrm{P}<0,001)$.

Com relação à espessura da patela, os grupos G1 e G2 poderiam se tornar uma única categoria de massa corporal, já que não houve diferença significativa em nenhuma das medidas realizadas nesses grupos. Porém, esses grupos mostraram diferença significativa nas medidas de comprimento interno e de largura interna da patela no terço proximal. Portanto, uma prótese é necessária para cada grupo, uma vez que ela é composta de medidas de comprimento, largura e espessura, e, consequentemente, não é possível fazer uma única prótese para G1 e G2.

Houve correlação forte e positiva entre as medidas internas e externas do comprimento da patela $(\mathrm{r}=0,87 ; \mathrm{P}<0,001)$ e entre a largura interna da patela no terço médio e a largura externa da patela no terço médio $(\mathrm{r}=0,86 ; \mathrm{P}<0,001)$. Com base nisso, determinou-se o comprimento e a largura interna da patela por meio das medidas externas, utilizando-se as fórmulas:

comprimento interno $=-0,0466+(0,751 \cdot$ comprimento externo);

largura interna $=0,177+(0,650 \cdot$ largura externa).

Esses resultados mostram que é possível utilizar a medida externa do comprimento e da largura da patela para estimar as medidas internas. Dessa forma, não seria necessária a artrotomia da articulação femorotibiopatelar para se determinarem as medidas internas da patela. Elas se complementam e adicionam informações úteis e necessárias para a confecção de prótese de patela segundo o grupo de massa corporal.

As análises estatísticas mostraram diferenças entre os cinco grupos, levando a concluir, portanto, que cada faixa de massa corporal tem suas medidas próprias. Não é possível fazer proporção entre as faixas de massa corporal. Como pode ser observado, há um aumento crescente nas medidas das patelas de acordo com as categorias de massa corporal dos grupos, porém não há uma constância na proporção de variação entre os grupos. Assim, não é possível aumentar ou diminuir o tamanho das próteses proporcionalmente a um modelo padrão. Existe também diferença numérica (milímetros) dentro do mesmo grupo, o que leva a questionamentos de ordem prática. Variações milimétricas podem ser importantes para congruência e funcionamento adequado da articulação (Dokic et al., 2015).

Estudos realizaram medidas nas patelas de cães normais (Yasukawa et al., 2016) e com LP (Towle et al., 2005; Yasukawa et al., 2016) para estudar as deformidades ósseas causadas por essa doença. Entretanto, esses autores realizaram um menor número de medidas do que as do presente trabalho. Yasukawa e colaboradores (2016) demonstraram que cães com luxação medial de patela possuem a patela com menor comprimento, largura e volume quando comparados a cães normais da mesma raça. Os autores sugerem que a incongruência entre a patela e o sulco troclear pode levar à hipoplasia 
patelar. Portanto, é importante conhecer as medidas em cães sem LP como no presente trabalho, uma vez que, segundo os autores acima mencionados, se essas medidas fossem realizadas em cães com LP, elas poderiam apresentar valores inferiores àqueles reais da patela.

Um fator que poderia interferir nos resultados do presente estudo seria as condições físicas dos cadáveres utilizados. Para amenizar esse viés, após inspeção visual, não foram utilizados cadáveres de cães que apresentavam características de animais obesos e caquéticos. A Tab. 3 mostra os dados das mensurações internas do sulco troclear nos grupos G1, G2, G3, G4 e G5. Com relação aos dados da mensuração da profundidade do sulco troclear no terço proximal do côndilo lateral, não houve diferença significativa entre os grupos G1 e G2. Os grupos G3 e G4 foram significativamente diferentes do G1 e do G2, mas não entre si. O grupo G5 foi significativamente diferente de todos os grupos.

Tabela 3. Média das medidas internas do sulco troclear de cadáveres de cães nos grupos G1, G2, G3, G4 e G5

\begin{tabular}{lccccc}
\hline \begin{tabular}{l} 
Mensurações internas do sulco \\
\multicolumn{1}{c}{ troclear }
\end{tabular} & $\mathrm{G} 1$ & $\mathrm{G} 2$ & $\mathrm{G} 3$ & $\mathrm{G} 4$ & $\mathrm{G} 5$ \\
\cline { 2 - 6 } & $0,10 \pm 0,01$ & $0,14 \pm 0,01$ & $0,24 \pm 0,01^{* \#}$ & $0,27 \pm 0,02^{* \#}$ & $0,36 \pm 0,02^{* \#+\S}$ \\
\hline $\begin{array}{l}\text { Profundidade no terço } \\
\text { proximal do côndilo lateral }\end{array}$ & $0,10 \pm 0,01$ & $0,13 \pm 0,01$ & $0,16 \pm 0,01^{*}$ & $0,20 \pm 0,01^{* \#}$ & $0,24 \pm 0,01^{* \#+}$ \\
$\begin{array}{l}\text { Profundidade no terço médio } \\
\text { do côndilo lateral }\end{array}$ & $0,09 \pm 0,006$ & $0,12 \pm 0,008$ & $0,12 \pm 0,008$ & $0,16 \pm 0,01^{*}$ & $0,21 \pm 0,01^{* \#+\S}$ \\
$\begin{array}{l}\text { Profundidade no terço distal } \\
\text { do côndilo lateral }\end{array}$ & $0,15 \pm 0,02$ & $0,16 \pm 0,01$ & $0,25 \pm 0,02^{*}$ & $0,31 \pm 0,02^{* \#}$ & $0,36 \pm 0,03^{* \#+}$ \\
$\begin{array}{l}\text { Profundidade no terço } \\
\text { proximal do côndilo medial }\end{array}$ & $0,12 \pm 0,01$ & $0,13 \pm 0,008$ & $0,16 \pm 0,01$ & $0,20 \pm 0,01^{* \#}$ & $0,25 \pm 0,02^{* \#+}$ \\
$\begin{array}{l}\text { Profundidade no terço médio } \\
\text { do côndilo medial }\end{array}$ & $0,10 \pm 0,009$ & $0,11 \pm 0,008$ & $0,13 \pm 0,009$ & $0,14 \pm 0,01$ & $0,18 \pm 0,01^{* \#+}$ \\
$\begin{array}{l}\text { Profundidade no terço distal } \\
\text { do côndilo medial }\end{array}$ & $0,47 \pm 0,01$ & $0,58 \pm 0,02$ & $0,80 \pm 0,04^{* \#}$ & $1,00 \pm 0,02^{* \#+}$ & $1,13 \pm 0,03^{* \#+\S}$ \\
$\begin{array}{l}\text { Largura do sulco troclear no } \\
\text { terço proximal }\end{array}$ & $0,44 \pm 0,01$ & $0,54 \pm 0,02$ & $0,70 \pm 0,03^{* \#}$ & $0,86 \pm 0,02^{* \#+}$ & $0,99 \pm 0,03^{* \#+\S}$ \\
$\begin{array}{l}\text { Largura do sulco troclear no } \\
\text { terço médio }\end{array}$ & $0,47 \pm 0,01$ & $0,53 \pm 0,01$ & $0,64 \pm 0,02^{* \#}$ & $0,79 \pm 0,02^{* \#+}$ & $0,89 \pm 0,02^{* \#+\S}$ \\
$\begin{array}{l}\text { Largura do sulco troclear no } \\
\text { terço proximal }\end{array}$ & & & & & \\
\hline
\end{tabular}

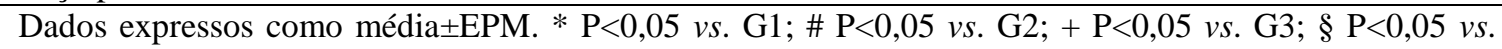
G4. $\uparrow$, aumento.

Houve uma correlação forte e positiva entre a massa corporal dos animais e a profundidade no terço proximal do côndilo lateral ( $\mathrm{r}=0,76$; $\mathrm{P}<0,001)$. Com relação à profundidade do sulco troclear no terço médio do côndilo lateral, não houve diferença significativa entre os grupos G1 e G2. O grupo G3 foi significativamente diferente do G1. O grupo G4 foi significativamente diferente dos grupos $\mathrm{G} 1$ e G2, mas não de G3 e G5. O grupo G5 apresentou diferença significativa dos grupos G1, G2 e G3. Houve uma correlação moderada e positiva entre a massa corporal dos animais e a profundidade do sulco troclear no terço médio do côndilo lateral $(\mathrm{r}=0,68 ; \quad \mathrm{P}<0,001)$. Com relação à profundidade do sulco troclear no terço distal do côndilo lateral, não houve diferença significativa entre os grupos G1, G2 e G3. O grupo G4 foi significativamente diferente do G1, e o grupo G5 apresentou diferença significativa de todos os grupos. Houve uma correlação moderada e positiva entre a massa corporal dos animais e a profundidade do sulco troclear no terço distal do côndilo lateral ( $\mathrm{r}=0,67 ; \mathrm{P}<0,001)$.

Com relação à profundidade do sulco troclear no terço proximal do côndilo medial, não houve diferença significativa entre os grupos G1 e G2. O grupo G3 foi significativamente diferente do G1. O grupo G4 foi significativamente diferente de G1 e G2, mas não de G3 e G5. O grupo G5 apresentou diferença significativa dos grupos G1, 
G2 e G3. Houve uma correlação moderada e positiva entre a massa corporal dos animais e a profundidade do sulco troclear no terço proximal do côndilo medial $(\mathrm{r}=0,62 ; \mathrm{P}<, 001)$. Em relação à profundidade do sulco troclear no terço médio do côndilo medial, não houve diferença significativa entre os grupos G1, G2 e G3. O grupo $\mathrm{G} 4$ foi significativamente diferente dos grupos G1 e G2, mas não de G3 e G5. O grupo G5 apresentou diferença significativa dos grupos G1, G2 e G3. Houve uma correlação moderada e positiva entre a massa corporal dos animais e a profundidade do sulco troclear no terço médio do côndilo medial $(\mathrm{r}=0,65 ; \mathrm{P}<0,001)$. Em relação à profundidade do sulco troclear no terço distal do côndilo medial, não houve diferença significativa entre os grupos G1, G2, G3 e G4. O grupo G5 apresentou diferença significativa dos grupos $\mathrm{G} 1$, G2 e G3, mas não de G4. Houve uma correlação moderada e positiva entre a massa corporal dos animais e a profundidade do sulco troclear no terço distal do côndilo medial ( $\mathrm{r}=0,48 ; \mathrm{P}<0,001)$.

A Tab. 3 também apresenta os dados referentes à largura do sulco troclear. Em relação à largura do sulco troclear no terço proximal, não houve diferença significativa entre os grupos G1 e G2. Os grupos G3, G4 e G5 foram significativamente diferentes dos grupos G1 e G2 e entre si. Houve uma correlação forte e positiva entre a massa corporal dos animais e a largura do sulco troclear no terço proximal $(\mathrm{r}=0,86 ; \mathrm{P}<0,001)$. Em relação à largura do sulco troclear no terço médio, não houve diferença significativa entre os grupos G1 e G2. Os grupos G3, G4 e G5 foram significativamente diferentes dos grupos G1 e G2 e entre si. Houve uma correlação forte e positiva entre a massa corporal dos animais e a largura do sulco troclear no terço médio $(\mathrm{r}=0,86 ; \mathrm{P}<0,001)$. Em relação à largura do sulco troclear no terço distal, assim como as medidas da largura do sulco nos terços proximal e médio, não houve diferença significativa entre os grupos G1 e G2. Os grupos G3, G4 e G5 foram significativamente diferentes dos grupos G1 e G2 e entre si. Houve uma correlação forte e positiva entre a massa corporal dos animais e a largura do sulco troclear no terço distal $(\mathrm{r}=0,86 ; \mathrm{P}<0,001)$. Todos esses resultados das medidas do sulco troclear são importantes, uma vez que a prótese irá se movimentar sobre esse sulco. A congruência entre essas duas estruturas é fundamental para o bom funcionamento dessa articulação (Dokic et $a l ., 2015)$. As medidas do sulco troclear não serão utilizadas para a confecção da prótese, entretanto elas podem subsidiar a necessidade de trocleoplastia, bem como a profundidade e a largura em que ela deve ser realizada para acomodar a prótese específica.

As próteses de patela já são utilizadas em humanos há algum tempo (Worrel, 1979), são confeccionadas com uma liga de cobalto-cromo e fixadas aos fragmentos remanescentes de patela por meio de polimetilmetacrilato (Worrel, 1979). $\mathrm{O}$ autor recomenda o uso de próteses patelares com ou sem componentes protéticos femorais. Atualmente existem diferentes marcas de próteses humanas disponíveis: Surgival $^{\circledR}$, Medacta $^{\circledR}$ e Zimmer Biomet $^{\circledR}$, porém, na literatura consultada, esse tipo de prótese ainda não está disponível para o uso na medicina veterinária. Do exposto, têm-se, portanto, teoricamente, valores médios de cada categoria de massa corporal para a confecção prévia da prótese de patela. Entretanto, estudos futuros devem ser realizados, com o objetivo de avaliar a funcionalidade das próteses confeccionadas com base nos valores médios dos cinco grupos.

\section{CONCLUSÕES}

Nas condições deste estudo, pode-se concluir que o tamanho da patela varia segundo a massa corporal do cão e que essas medidas devem ser consideradas ao se planejar uma substituição protética. As medidas externas da patela podem ser usadas para estimar o tamanho desse osso sesamoide e auxiliar na confecção de prótese de patela de cão.

\section{AGRADECIMENTOS}

À Escola de Veterinária da UFMG, ao Conselho Nacional de Desenvolvimento Científico e Tecnológico (CNPq), Coordenação de Aperfeiçoamento de Pessoal de Nível Superior (CAPES) e à Fundação de Amparo à Pesquisa do Estado de Minas Gerais (Fapemig) pelo apoio à pesquisa. 


\section{REFERÊNCIAS}

CAMPBELL, J.R.; POND, M.J. The canine stifle joint- Medial luxation of the patella. Small. Anim. Pract., v.13, p.11-18, 1972.

CARPENTER JR, D.H.; COOPER, R.C. Mini review of canine stifle joint anatomy. Anat. Histol. Embryol., v.29, p.321-329, 2000.

DAEMS, R.; JANSSENS, L.A.; BÉOSIER, Y.M. Grossly apparent cartilage erosion of the patellar articular surface in dogs with congenital medial patellar luxation. Vet. Comp. Orthop. Traumatol., v.3, p.222-224, 2009.

DOKIC, Z.; LORINSON, D.; WEIGEL, J.P. et al. Patellar groove replacement in patellar luxation with severe femoro-patellar osteoarthritis. Vet. Comp. Orthop. Traumatol., v.2, p.124-130, 2015.

EVANS, H.E.; LAHUNTA, A. The skeleton. In: EVANS, H.E., LAHUNTA, A. (Eds.). Miller's anatomy of the dog. 4.ed. St. Louis, Missouri: Elsevier, 2013. p.44-148.

GARETH, I.A.; SORREL, J.L.H. Complications associated with corrective surgery for patellar luxation in 109 dogs. Vet. Surg., v.35, p.559-566, 2006.

KOWALESKI M.P.; BOUDRIEAU, R.J.; POZZI, A. Stifle joint. In: TOBIAS, K.; JOHNSTON, S. (Eds.). Veterinary surgery small animal.. St. Louis, Missouri: Elsevier, 2012. p.906-999.

LAFOND, E.; BREUR, G.J.; AUSTIN, C.C. Breed susceptibility for developmental orthopedic diseases in dogs. J. Am. Anim. Hosp. Assoc., v.38, p.467-477, 2002.

LARA, J.S. Caracterização dos aspectos clínicos, epidemiológicos e lesões associadas à luxação de patela em cães atendidos no hospital veterinário no período de 2000 a 2010: estudo retrospectivo. 2011. 56f. Dissertação (Mestrado em Medicina Veterinária) - Escola de Veterinária, Universidade Federal de Minas Gerais, Belo Horizonte, MG.
LIEBICH, H.G.; KÖNIG, H.E.; MAIERL, J. Membros pélvicos. In: LIEBICH, H.G.; KÖNIG, H.E. (Eds.). Anatomia dos animais domésticos. 6.ed. Porto Alegre: Artmed, 2016. p.233-238.

MCKEE, M.W.; COOK, J.L. The stifle. In: HOULTON, J.E.E.; COOK, J.L.; INNES, J.F. et al. (Eds.). BSAVA manual of canine and feline musculoskeletal disorders. Gloucester: BSAVA, 2006. p.350-374.

O'NEILL, D.G.; MEESON, R.L.; SHERIDAN, A. et al. The epidemiology of patellar luxation in dogs attending primary-care veterinary practices in England. Can. Genet. Epid., v.3, p.1-12, 2016.

PIERMATTEI, D.L.; FLO, G.L.; DECAMP, C.E. The stifle joint. In: PIERMATTEI, D.L.; FLO, G.L.; DECAMP, C.E. (Eds.). Small animal orthopedics and fracture repair. 4.ed. Philadelphia: Saunders, 2006. p.637-685.

REZENDE, C.M.F.; TÔRRES, R.C.S.; NEPOMUCENO, A.C. et al. Patellar luxation in small animals. In: KAOUD, H.A.E. (Eds.). Canine medicine - recent topics and advanced research. London: InTEC, 2016. p.159-178.

ROUSH, J.K. Canine patellar luxation. Vet. Clin. N. Am. Small Anim. Pract., v.23, p.855-868, 1993.

TOWLE, H.A.; GRIFFON, D.J.; THOMAS, M.W. et al. Pre and postoperative radiographic and computed tomographic evaluation of dogs with medial patellar luxation. Vet. Surg., v.34, p.265-272, 2005.

WILLAUER, C.C.; VASSEUR, P.B. Clinical results of surgical correction of medial luxation of the patella in dogs. Vet. Surg., v.16, p.31-36, 1987.

WORRELL, R.V. Prosthetic resurfacing of the patella. Clin. Orthop. Relat. Res., v.144, p.91-97, 1979.

YASUKAWA, S.; EDAMURA, K.; TANEGASHIMA, K. et al. Evaluation of bone deformities of the femur, tibia, and patella in Toy Poodles with medial patellar luxation using computed tomography. Vet. Comp. Orthop. Traumatol., v.1, p.29-38, 2016. 